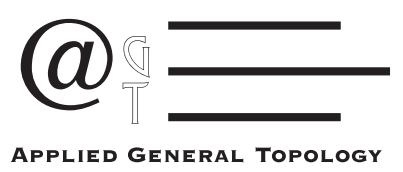

\title{
Fuzzy Uniformities on Function Spaces
}

\author{
J. K. Kohli and A. R. Prasannan*
}

\begin{abstract}
We study several uniformities on a function space and show that the fuzzy topology associated with the fuzzy uniformity of uniform convergence is jointly fuzzy continuous on $C_{f}(X, Y)$, the collection of all fuzzy continuous functions from a fuzzy topological space $X$ into a fuzzy uniform space $Y$. We define fuzzy uniformity of uniform convergence on starplus-compacta and show that its corresponding fuzzy topology is the starplus-compact open fuzzy topology. Moreover, we introduce the notion of fuzzy equicontinuity and fuzzy uniform equicontinuity on fuzzy subsets of a function space and study their properties.
\end{abstract}

2000 AMS Classification: 03E72, 04A72, 54A40, 54C35, 54D30, 54E15.

Keywords: Starplus-compact open fuzzy topology, fuzzy uniformity of uniform convergence, jointly fuzzy continuous fuzzy topology, fuzzy uniformity of uniform convergence on starplus-compacta, fuzzy equicontinuity, fuzzy uniform equicontinuity.

\section{INTRODUCTION}

The notion of a uniform space was introduced by Andre Weil [18] in 1937. The first systematic exposition of the theory of uniform spaces was given by Bourbaki [4] in 1940. Weil elaborated the topology associated with a uniformity and proved that a topological space is uniformizable if and only if it is completely regular. He extended the notion of uniform continuity and uniform isomorphisms to the framework of uniform spaces and obtained the uniform space version of Alexandroff-Uryshon metrization theorem that a uniform space is metrizable if and only if its uniformity has a countable base. The concept of function space was evolved around the close of the nineteenth century and the study of function spaces began with the work of Ascoli [3], Arzelà [2] and Hadamard [8]. The uniformity of pointwise convergence and uniform

${ }^{*}$ Corresponding author. 
convergence were first defined and studied by Fox [7]. The term function space is introduced much earlier in connection with questions of a topological nature about sets of functions. The study of topologies on function spaces is an active area of research and, besides their multifaceted applications, forms a well-established and sound body of knowledge.

The study of useful fuzzy topologies and uniformities on function spaces, besides their intrinsic interest, is important from applications viewpoint. The first effort in this direction was made by Peng [17] in 1984. Subsequently, Alderton [1] studied the problem from categorical viewpoint and utilized the well-developed theory of cartesian closedness of monotopological categories to the fuzzy topologies on a function space. Burton [6] defined analogues of the uniformities of pointwise convergence and uniform convergence and obtained conditions for completeness and compactness of fuzzy subsets of a function space. Jäger considered fuzzy uniform convergence and equicontinuity in [9]. In [13], we defined three different fuzzy topologies on a function space, which are analogues of the topology of pointwise convergence, compact-open topology and the topology of joint continuity in general topology.

In this paper, we elaborate on the pointwise fuzzy uniformity, fuzzy uniformity of uniform convergence and fuzzy uniformity of uniform convergence on starplus compacta and their associated fuzzy topologies on a function space. It turns out that the fuzzy topology associated with the fuzzy uniformity of uniform convergence is jointly continuous; and that the fuzzy topology of uniform convergence on starplus compacta is the starplus-compact open fuzzy topology [13]. Further, we study the notion of fuzzy equicontinuity on fuzzy subsets of a family of functions from a fts/fuzzy uniform space to a fuzzy uniform space.

\section{Preliminaries}

Throughout the paper the closed unit interval $[0,1]$ will be denoted by $I$. The symbols and $I_{0}$ and $I_{1}$ will stand for the intervals $(0,1]$ and $[0,1)$, respectively.

Definition 2.1. For a fuzzyy set $\mu$ in $X$, the set $\mu^{\alpha}=\{x \in X: \mu(x)>\alpha\}$ and $\mu_{\alpha}=\{x \in X: \mu(x) \geq \alpha\}$ are called the strong $\alpha$-level set of $\mu$ and the weak $\alpha$-level set of $\mu$, respectively. The set $\{x \in X: \mu(x)>0\}$ is called the support of $\mu$ and is denoted by supp $\mu$.

Definition 2.2 ([13]). A fuzzy set $\mu$ in a fts $(X, \tau)$ is said to be starpluscompact if $\mu^{\alpha}$ is compact in $\left(X, i_{\alpha}(\tau)\right)$ for each $\alpha \in I_{1}$. The fts $(X, \tau)$ is said to be starplus-compact if $\left(X, i_{\alpha}(\tau)\right)$ is compact for each $\alpha \in I_{1}$.

Let $\mathrm{X}$ be a non-empty set and let $(X, \tau)$ be a fuzzy topological space. Let $Y^{X}$ denote the collection of all functions from $X$ into $Y$ and let $\Im$ be a nonempty subset of $Y^{X}$.

Definition 2.3 ([12]). For each $x \in X$, let the map $e_{x}: \Im \longrightarrow Y$ be defined by $e_{x}(f)=f(x)$. We call $e_{x}$ the evaluation map at $x \in X$. The initial fuzzy topology on $\Im$ generated by the collection of maps $\left\{e_{x}: x \in X\right\}$ is called the 
pointwise fuzzy topology on $\Im$ and is denoted by $\tau_{p}$. The pair $\left(\Im, \tau_{p}\right)$ is called the pointwise fuzzy function space.

The pointwise fuzzy topology on $\Im$ concides with the subspace fuzzy topolology it inherits as a subspace of the product fuzzy topology on $Y^{X}$. .

Definition $2.4([13])$. Let $(X, \tau)$ and $(Y, \sigma)$ be fts and let $\Im$ be a nonempty subset of $Y^{X}$. For each starplus-compact fuzzy set $\kappa$ in $X$ and each open fuzzy set $\mu$ in $Y$, define a fuzzy set $\kappa_{\mu}$ on $\Im$ by $\kappa_{\mu}=\bigwedge_{x \in \text { supp }} e_{x}^{-1}(\mu)$. The collection of all fuzzy sets $\kappa_{\mu}$, where $\kappa$ is a starplus-compact fuzzy set in $X$ and $\mu$ is an open fuzzy set in $Y$, forms a subbase for a fuzzy topology $\tau_{* C}^{+}$on $\Im$ called the starplus-compact open fuzzy topology on $\Im$. The pair $\left(\Im, \tau_{* C}^{+}\right)$is referred to as a starplus-compact open fuzzy function space.

Proposition 2.5 ([13]). The starplus-compact open fuzzy topology on $\Im$ is stronger than the pointwise fuzzy topology $\tau_{p}$ on $\Im$.

Theorem 2.6 ([13]). Let $\left(X, \tau_{X}\right)$ be a topologically generated fts and let $\left(Y, \tau_{Y}\right)$ be a fts. Then a fuzzy topology $\tau$ is a starplus-compact open fuzzy topology on $\Im$ if and only if $i_{\alpha}(\tau)=T_{C}^{\alpha}$ for each $\alpha \in I_{1}$, where $T_{C}^{\alpha}$ denotes the compact open topology on $\Im$ and $X$ is endowed with the topology $i_{0}\left(\tau_{X}\right)$ and $Y$ is equipped with the topology $i_{\alpha}\left(\tau_{Y}\right)$.

Definition 2.7 ([13]). A fuzzy topology $\tau$ on $\Im$ such that the map $\phi: \Im \times X \longrightarrow$ $Y$ defined by $\phi(f, x)=f(x)$ is fuzzy continuous, where $\Im \times X$ is endowed with the product fuzzy topology, is called a jointly fuzzy continuous fuzzy topology on $\Im$.

Theorem 2.8 ([13]). The fuzzy topology of joint fuzzy continuity is a good extension.

Definition 2.9 ([15]). A subset $\mathcal{F} \subset I^{X}$ is called a prefilter if and only if $\mathcal{F} \neq \phi$, and

i) For all $\mu, \nu \in \mathcal{F}$ we have $\mu \wedge \nu \in \mathcal{F}$.

ii) If $\mu \geq \nu$ and $\nu \in \mathcal{F}$, then $\mu \in \mathcal{F}$.

iii) $\mathbf{0} \notin \mathcal{F}$.

Definition 2.10 ([15]). A subset $\mathcal{B} \subset I^{X}$ is a base for a prefilter if and only if $\mathcal{B} \neq \phi$, and

i) For all $\mu, \nu \in \mathcal{B}$ there exists a $\xi \in \mathcal{B}$ such that $\xi \leq \mu \wedge \nu$.

ii) $\mathbf{0} \notin \mathcal{B}$.

Definition 2.11 ([15]). A prefilter generated by a prefilter base $\mathcal{B}$ is denoted as $\langle\mathcal{B}\rangle$ and $\langle\mathcal{B}\rangle=\left\{\mu \in I^{X}\right.$ : there exists a $\nu \in \mathcal{B}$ such that $\left.\mu \geq \nu\right\}$. If $\mathcal{B}$ is a prefilter base, then $\widehat{\mathcal{B}}=\left\{\sup _{\epsilon \in I_{0}}\left(\beta_{\epsilon}-\epsilon\right):\left(\beta_{\epsilon}\right)_{\epsilon \in I_{0}} \in \mathcal{B}^{I_{0}}\right\}$.

Proposition $2.12([15])$. If $\mathcal{B}$ is a prefilter base, then $\langle\widehat{\mathcal{B}}\rangle=\langle\mathcal{B}\rangle$. We shall denote by $\widetilde{\mathcal{B}}$ the prefilter $\langle\widehat{\mathcal{B}}\rangle=\langle\mathcal{B}\rangle$. 
Definition 2.13 ([15]). A prefilter $\mathcal{F}$ is called prime if $\mu \vee \nu \in \mathcal{F}$ implies $\mu \in \mathcal{F}$ or $\nu \in \mathcal{F}$.

Definition 2.14 ([15]). If $\mathcal{F}$ is a prefilter on $X$, then we define the following: $P(\mathcal{F})=\{\mathcal{G}: \mathcal{G}$ is a prime prefilter and $\mathcal{F} \subset \mathcal{G}\}$ and $P_{m}(\mathcal{F})=\{\mathcal{G}: \mathcal{G} \in P(\mathcal{F})$ and $\mathcal{G}$ is minimal $\}$.

Definition $2.15([15])$. For a prefilter $\mathcal{F}$ the characteristic of $\mathcal{F}$ is defined by $\mathbf{c}(\mathcal{F})=\inf _{\nu \in \mathcal{F}} \sup \nu$. For a prefilter $\mathcal{F}$ the lower characteristic of $\mathcal{F}$ is defined by $\overline{\mathbf{c}}(\mathcal{F})=\inf _{\mathcal{G} \in P_{m}(\mathcal{F})} \mathbf{c}(\mathcal{G})$. If $\mathcal{F}$ is a prime, then $\overline{\mathbf{c}}(\mathcal{F})=\mathbf{c}(\mathcal{F})$.

Definition 2.16 ([16]). If $X$ is a set, $\mu \in I^{X}$ and $\nu \in I^{X \times X}$, then the section of $\nu$ over $\mu$ is defined by $\nu\langle\mu\rangle(x)=\sup _{y \in X} \mu(y) \wedge \nu(y, x)$ for all $x \in X$.

Definition $2.17([16])$. If $\mu, \nu \in I^{X \times X}$, then the composition $\mu \circ \nu$ is defined by $\mu \circ \nu(x, y)=\sup _{z \in X} \nu(x, z) \wedge \mu(z, y)$ for all $(x, y) \in X \times X$.

Definition 2.18 ([16]). If $\nu \in I^{X \times X}$, then its symmetric ${ }_{s} \nu \in I^{X \times X}$ is defined by ${ }_{s} \nu(x, y)=\nu(y, x)$ for all $(x, y) \in X \times X$.

Throughout this paper we follow the terminology and notions of a fuzzy uniformity as defined by Lowen [16].

Definition 2.19 ([16]). A fuzzy uniformity on $X$ is a subset $\mathbf{U} \subset I^{X \times X}$, which satisfies the following conditions:

i) $\mathbf{U}$ is a prefilter.

ii) $\widehat{\mathbf{U}}=\mathbf{U}$, i.e., for every family $\left(\nu_{\epsilon}\right)_{\epsilon \in I_{0}} \in \mathbf{U}^{I_{0}} \Longrightarrow \sup _{\epsilon \in I_{0}}\left(\nu_{\epsilon}-\epsilon\right) \in \mathbf{U}$.

iii) For all $\nu \in \mathbf{U}$ and for all $x \in X, \nu(x, x)=1$.

iv) For all $\nu \in \mathbf{U},{ }_{s} \nu \in \mathbf{U}$.

v) For all $\nu \in \mathbf{U}$ and for all $\epsilon \in I_{0}$ there exists $\nu_{\epsilon} \in \mathbf{U}$ such that $\nu_{\epsilon} \circ \nu_{\epsilon}-\epsilon \leq$ $\nu$.

The pair $(X, \mathbf{U})$ is called a fuzzy uniform space.

Definition 2.20 ([16]). A subset $\mathcal{B} \subset I^{X \times X}$ is called a base for a fuzzy uniformity if and only if the following conditions hold:

i) $\mathcal{B}$ is a prefilter basis.

ii) For all $\beta \in \mathcal{B}$ and for all $x \in X, \beta(x, x)=1$.

iii) For all $\beta \in \mathcal{B}$ and for all $\epsilon \in I_{0}$, there exists $\beta_{\epsilon} \in \mathcal{B}$ such that $\beta_{\epsilon}-$ $\epsilon \leq{ }_{s} \beta$.

iv) For all $\beta \in \mathcal{B}$ and for all $\epsilon \in I_{0}$, there exists $\beta_{\epsilon} \in \mathcal{B}$ such that $\beta_{\epsilon} \circ \beta_{\epsilon}-$ $\epsilon \leq \beta$.

Definition 2.21. If $\mathbf{U}$ is a fuzzy uniformity on $X$ then $\mathcal{B} \subset I^{X \times X}$ is a basis for $\mathbf{U}$ iff $\mathcal{B}$ is a prefilter basis and $\widetilde{\mathcal{B}}=\mathbf{U}$.

Proposition 2.22. If $\mathbf{U}$ is a fuzzy uniformity on $X$, then the family of symmetric fuzzy entourages ${ }_{s} \mathbf{U}=\left\{\nu \in \mathbf{U}:{ }_{s} \nu=\nu\right\}$ is a basis for $\mathbf{U}$. 
Definition 2.23. Let $(X, \tau)$ be a fts. Then the closure $\bar{\mu}$ of a fuzzy set $\mu$ of $X$ is defined as $\bar{\mu}=\inf \{\nu: \mu \leq \nu, 1-\nu \in \tau\}$.

Definition 2.24 ([14]). A fuzzy closure operator on a fts $X$ is a map ${ }^{-}: I^{X} \longrightarrow I^{X}$ which satisfies the following conditions:

i) $\bar{\alpha}=\alpha$, for all $\alpha \in I$.

ii) $\bar{\mu} \geq \mu$, for all $\mu \in I^{X}$.

iii) $\overline{\mu \vee \nu}=\bar{\mu} \vee \bar{\nu}$, for all $\mu, \nu \in I^{X}$.

iv) $\overline{\bar{\mu}}=\bar{\mu}$, for all $\mu \in I^{X}$.

Proposition 2.25 ([16]). Let $(X, U)$ be a fuzzy uniform space. The map ${ }^{-}: I^{X} \longrightarrow I^{X}$ defined by $\bar{\mu}=\inf _{\nu \in \mathbf{U}} \nu\langle\mu\rangle$ is a fuzzy closure operator.

Definition 2.26 ([5]). If $\mathcal{F}$ is a prefilter on $(X, \mathbf{U})$, then $A d h \mathcal{F}$ and $\lim \mathcal{F}$ are fuzzy sets in $X$ and is defined by $A d h \mathcal{F}=\inf _{\nu \in \mathcal{F}} \bar{\nu}$ and $\lim \mathcal{F}=\inf _{\mathcal{G} \in \mathbf{P}_{\mathbf{m}}(\mathcal{F})}$ Adh $\mathcal{G}$.

If $\mu \in I^{X}$, we say that $\mathcal{F}$ is $\mathbf{U}$-convergent in $\mu$ iff $\bar{c}(\mathcal{F}) \leqslant \sup \mu \wedge \lim \mathcal{F}$ and $\mathcal{F}$ is $\mathbf{U}$-convergent iff $\bar{c}(\mathcal{F}) \leqslant \sup \lim \mathcal{F}$.

Definition 2.27. Let $(X, \mathbf{U})$ be a fuzzy uniform space and $\mathcal{F}$ is a prefilter on $X$. Then $\mathcal{F}$ is $\mathbf{U}$-cauchy iff $c(\mathcal{F}) \leqslant \inf _{\sigma \in \mathbf{U}} \sup \inf _{\mathcal{G} \in \mathbf{P}_{\mathbf{m}}(\mathcal{F})} \inf _{\nu \in \mathcal{G}} \sigma\langle\nu\rangle$.

Definition 2.28. Let $(X, \mathbf{U})$ and $\left(Y, \mathbf{U}^{1}\right)$ be fuzzy uniform spaces. Then a map $f: X \longrightarrow Y$ is said to be fuzzy uniformly continuous if for each $\nu \in \mathbf{U}^{1}$, $(f \times f)^{-1}(\nu) \in \mathbf{U}$.

Proposition 2.29. If $(X, \mathbf{U})$ and $\left(Y, \mathbf{U}^{1}\right)$ are fuzzy uniform spaces, $\mathcal{B}$ and $\mathcal{B}^{1}$ are basis for $\mathbf{U}$ and $\mathbf{U}^{1}$, respectively and $f: X \longrightarrow Y$, then $f$ is fuzzy uniformly continuous iff for all $\beta^{1} \in \mathcal{B}^{1}$ and for all $\varepsilon \in I_{0}$ there exist $\beta \in \mathcal{B}$ such that $\beta-\varepsilon \leq(f \times f)^{-1}\left(\beta^{1}\right)$.

Theorem 2.30. If $(X, \mathbf{U})$ and $\left(Y, \mathbf{U}^{1}\right)$ are fuzzy uniform space and $f: X \longrightarrow$ $Y$ is uniformly continuous, then $f$ is fuzzy continuous.

Throughout this paper uniformity on a nonempty set $X$ is denoted by $\mathcal{U}$ and a fuzzy uniformity by $\mathbf{U}$. We denote the topology associated with a uniformity $\mathcal{U}$ by $T(\mathcal{U})$ and the fuzzy topology associated with a fuzzy uniformity $\mathbf{U}$ by $\tau(\mathbf{U})$ [16], where $\tau(\mathbf{U})$ is the fuzzy topology whose fuzzy closure operator is defined in Proposition 2.25.

Definition 2.31 ([16]). Let $X$ be a non empty set and let $\left\{f_{j}: X \rightarrow\left(Y_{j}, \mathcal{U}_{j}\right)\right.$, $j \in J\}$ be a family of functions from $X$ into the family of fuzzy uniform spaces $\left\{\left(Y_{j}, \mathcal{U}_{j}\right), j \in J\right\}$. Then the coarsest fuzzy uniformity $\mathbf{U}$ on $X$ making each $f_{j}, j \in J$ is fuzzy uniformly continuous is called the initial fuzzy uniformity on $X$ and is denoted by $\sup _{j \in J}\left(f_{j} \times f_{j}\right)^{-1}\left(\mathbf{U}_{j}\right)$.

Let UNIF denote the category of uniform spaces and uniformly continuous functions and FUNIF denote the category of fuzzy uniform spaces and fuzzy uniformly continuous functions. Then the functors $\omega_{u}:$ UNIF $\rightarrow$ FUNIF and 
$i_{u}:$ FUNIF $\rightarrow$ UNIF are defined as follows and are the uniform analogues of the functors $\omega$ and $i$ introduced in [14].

For each $(X, \mathcal{U}) \in \mathbf{U N I F}, \omega_{u}(X, \mathcal{U})=\left(X, \omega_{u}(\mathcal{U})\right)$, where $\omega_{u}(\mathcal{U})=\{\mu \in$ $\left.I^{X \times X}: \mu^{-1}(\alpha, 1] \in \mathcal{U}, \forall \alpha \in I_{1}\right\}$ and for each $(X, \mathbf{U}) \in \mathbf{F U N I F}, i_{u}(X, \mathbf{U})=$ $\left(X, i_{u}(\mathbf{U})\right)$, where $i_{u}(\mathbf{U})=\left\{\mu^{-1}(\alpha, 1]: \mu \in \mathbf{U}, \alpha \in I_{1}\right\}$.

Theorem 2.32 ([16]). If $\mathcal{U}$ is a uniformity on $X$ and $\mathbf{U}$ a fuzzy uniformity on $X$. Then,

1) $\omega_{u}(\mathcal{U})$ is a fuzzy uniformity on $X$.

2) $i_{u}(\mathbf{U})$ is a uniformity on $X$.

3) $i_{u}\left(\omega_{u}(\mathcal{U})\right)=\mathcal{U}$.

4) $\omega_{u}\left(i_{u}(\mathbf{U})\right)$ is the coarsest fuzzy uniformity generated by a uniformity and is finer than $\mathbf{U}$. We denote $\omega_{u}\left(i_{u}(\mathbf{U})\right)$ by $\overline{\mathbf{U}}$.

5) $\tau\left(\omega_{u}(\mathcal{U})\right)=\omega(T(\mathcal{U}))$

6) $T\left(i_{u}(\mathbf{U})\right)=i(\tau(\mathbf{U})$.

7) $\tau(\overline{\mathbf{U}})=\overline{\tau(\mathbf{U})}$.

We shall call a notion in FUNIF a good extension of a notion in UNIF if it reduces to the standard notion in case of the fuzzy uniformity $\mathbf{U}=\omega(\mathcal{U})$.

Definition 2.33 ([10]). If $\mathbf{U}$ is a fuzzy uniformity on a nonempty set $X$, then its $\alpha$-level uniformity, for $0 \leq \alpha \leq 1$ is defined by $i_{u, \alpha}(\mathbf{U})=\left\{\mu^{\beta} \in 2^{X \times X}\right.$ : $\mu \in \mathbf{U}, \beta \in[0,1-\alpha)\}$.

The functor $i_{u, \alpha}: \mathbf{F U N I F} \longrightarrow \mathbf{U N I F}$ is the uniform analogue of the functor $i_{\alpha}$ discussed in [16]. Also, $i_{u}=i_{u, 0}$.

Theorem 2.34 ([10]). The topology $T\left(i_{u, \alpha}(\mathbf{U})\right)$ on $X$, induced by the $\alpha$-level uniformity $i_{u, \alpha}(\mathbf{U})$ of the fuzzy uniform space $(X, \mathbf{U})$, coincides with the $\alpha$-level topology $i_{\alpha}(\tau(\mathbf{U}))$.

\section{Fuzzy Uniformities on Function Spaces}

Let $X$ be a non-empty set and let $(Y, \mathbf{U})$ be a fuzzy uniform space. Let $Y^{X}$ denote the collection of maps from $X$ into $Y$. Let $\Im$ be a nonempty subset of $Y^{X}$. In this section we study the pointwise fuzzy uniformity and fuzzy uniformity of uniform convergence and their associated fuzzy topologies. It is shown that the fuzzy topology associated with the fuzzy uniformity of uniform convergence is jointly fuzzy continuous on $C_{f}(X, Y)$.

Definition 3.1. The initial fuzzy uniformity $\mathbf{U}_{p}$ on $\Im$ generated by the collection of maps $\left\{e_{x}: x \in X\right\}$ is called the pointwise fuzzy uniformity or fuzzy uniformity of pointwise convergence on $\Im$. The pair $\left(\Im, \mathbf{U}_{p}\right)$ is called the pointwise fuzzy uniform space.

Remark 3.2. The above definition of pointwise fuzzy uniformity on $\Im$ coincides with the definitions of pointwise fuzzy uniformity given in $[6,9]$. 
The following theorem of Lowen [16] reflects upon the relationship that exists between the initial fuzzy topology and the fuzzy topology generated by the initial fuzzy uniformity.

Theorem 3.3 ([16]). Let $X$ be a set and let $\left(Y, \mathbf{U}_{j}\right), j \in J$ be a family of fuzzy uniform spaces. If $\left\{f_{j}: X \longrightarrow Y_{j}, j \in J\right\}$ is a family of functions, then $\tau\left(\sup _{j \in J}\left(f_{j} \times f_{j}\right)^{-1}\left(\mathbf{U}_{j}\right)\right)=\sup _{j \in J} f_{j}^{-1}\left(\tau\left(\mathbf{U}_{j}\right)\right)$.

In view of Definitions 2.3, 3.1 and Theorem 3.3, the following result is immediate.

Theorem 3.4. The fuzzy topology associated with the fuzzy uniformity $\mathbf{U}_{p}$ of pointwise fuzzy uniformity is the pointwise fuzzy topology $\tau_{P}$.

Theorem 3.5. Let $X$ be a non-empty set and let $(Y, \mathcal{U})$ be a uniform space. Let $\mathcal{U}_{p}$ denote the pointwise uniformity on $\Im$ and let $\mathbf{U}_{p}$ be the pointwise fuzzy uniformity on $\Im$, where $Y$ is endowed with the fuzzy uniformity $\omega_{u}(\mathcal{U})$. Then $\omega_{u}\left(\mathcal{U}_{p}\right)=\mathbf{U}_{p}$.

Proof. Let $\bigwedge_{i=1}^{n}\left(e_{x} \times e_{x}\right)^{-1}\left(\nu_{i}\right)$ be a basic element for the fuzzy uniformity $\mathbf{U}_{p}$, where $Y$ is endowed with the fuzzy uniformity $\omega_{u}(\mathcal{U})$. Since $\nu_{i} \in \omega_{u}(\mathcal{U}), \nu_{i}^{\alpha} \in$ $\mathcal{U}$ for each $\alpha \in I_{1}$ and hence $\left[\bigcap_{i=1}^{n}\left(e_{x} \times e_{x}\right)^{-1}\left(\nu_{i}^{\alpha}\right)\right] \in \mathcal{U}_{p}$. This shows that $\left[\bigwedge_{i=1}^{n}\left(e_{x} \times e_{x}\right)^{-1}\left(\nu_{i}\right]^{\alpha} \in \mathcal{U}_{p}\right.$ and so $\bigwedge_{i=1}^{n}\left(e_{x} \times e_{x}\right)^{-1}\left(\nu_{i}\right) \in \omega_{u}\left(\mathcal{U}_{p}\right)$. Thus we have $\omega_{u}\left(\mathcal{U}_{p}\right) \supseteq \mathbf{U}_{p}$. The proof of the opposite inclusion, $\omega_{u}\left(\mathcal{U}_{p}\right) \subseteq \mathbf{U}_{p}$, is similar to the one given above.

Proposition 3.6. Let $X$ be a set and let $(Y, \mathbf{U})$ be a fuzzy uniform space. Let $\mathbf{U}_{p}$ be the fuzzy uniformity of pointwise convergence on $\Im$. Then for each $\alpha \in I_{1}$, the $\alpha$-level uniformity $i_{u, \alpha}\left(\mathbf{U}_{p}\right)$ is the uniformity of pointwise convergence on $\Im$ with respect to $i_{u, \alpha}(\mathbf{U})$ on $Y$.

Proof. Let $\left(e_{x} \times e_{x}\right)^{-1}(\nu)$ be a subbasic element in $\mathbf{U}_{p}$.

Then for each $\alpha \in I_{1}$,

$$
\begin{aligned}
{\left[\left(e_{x} \times e_{x}\right)^{-1}(\nu)\right]^{\beta} } & =\{(f, g) \in \Im \times \Im: \nu(f(x), g(x))>\beta\}, \beta \in[0,1-\alpha) \\
& =\left\{(f, g) \in \Im \times \Im:(f(x), g(x)) \in \nu^{\beta}\right\} \\
& =\left(e_{x} \times e_{x}\right)^{-1}\left(\nu^{\beta}\right)
\end{aligned}
$$

which is a subbasic element in the pointwise uniformity on $\Im$, where $Y$ is endowed with the uniformity $i_{u, \alpha}(\mathbf{U})$.

Theorem $3.7([6])$. Let $\mathcal{F}$ be a prefilter in the pointwise fuzzy uniform space $\left(Y^{X}, \mathbf{U}_{p}\right)$. Then $\mathcal{F}$ is a Cauchy prefilter if and only if for each $x \in X, e_{x}(\mathcal{F})$ is a Cauchy prefilter in $\left(Y, \mathbf{U}_{Y}\right)$. 


\section{Fuzzy Uniformity of Uniform Convergence.}

In this subsection we study the notion of fuzzy uniformity of uniform convergence on a function space $\Im \subset Y^{X}$ (which was initiated by Burton [6]), where $X$ is a nonempty set and $(Y, \mathbf{U})$ is a fuzzy uniform space.

Definition $3.8([6])$. For each $\nu \in \mathbf{U}$, the fuzzy set $W_{\nu}=\bigwedge_{x \in X}\left(e_{x} \times e_{x}\right)^{-1}(\nu)$ in $\Im \times \Im$, where $e_{x}: \Im \longrightarrow Y$ is the evaluation map, is defined by,

$W_{\nu}(f, g)=\bigwedge_{x \in X} \nu(f(x), g(x))$. Let $\mathcal{B}$ be the collection of all $W_{\nu}$, where $\nu$ varies over $\mathbf{U}$.

Proposition $3.9([6])$. The collection $\mathcal{B}$ is a base for a fuzzy uniformity on $\Im$.

Example 3.10. If $\mu \in I^{\Im}$, then the section of $\mathrm{W}_{\nu}$ over $\mu$ is defined by

$$
\begin{aligned}
\mathrm{W}_{\nu}\langle\mu\rangle(f) & =\bigwedge_{x \in X} \nu\left\langle e_{x}(\mu)\right\rangle(f(x)) \\
& =\inf _{x \in X}\left\{\sup _{g \in \Im}\left(e_{x}(\mu)(g(x)) \bigwedge \nu(g(x), f(x))\right)\right\}
\end{aligned}
$$

for each $f \in \Im$.

Definition 3.11 ([6]). The fuzzy uniformity $\mathbf{U}_{u}$ generated by $\mathcal{B}$ is called the fuzzy uniformity of uniform convergence. The fuzzy topology associated with $\mathbf{U}_{u}$ is called the fuzzy topology of uniform convergence and it is denoted by $\tau_{u}$.

In the following results we give a short description of the concepts that Burton [6] uses relative to the fuzzy uniform convergence.

Theorem 3.12 $([6])$. Let $\mathcal{F}$ be a prefilter in $Y^{X}$ with $\mathbf{c}(\mathcal{F})=\overline{\mathbf{c}}(\mathcal{F})$. Then $\mathcal{F}$ is $\mathbf{U}_{u}$-cauchy, $\alpha \leq \overline{\mathbf{c}}, \lim _{\mathbf{U}_{p}}(\mathcal{F})(f) \geq \alpha \Longrightarrow \lim _{\mathbf{U}_{u}}(\mathcal{F})(f) \geq \alpha$.

Theorem $3.13([6])$. If $(X, \mathbf{U})$ is complete then $\left(Y^{X}, \mathbf{U}_{\mathbf{u}}\right)$ is complete.

Corollary $\mathbf{3 . 1 4}([6])$. If $\mu: Y^{X} \longrightarrow I$ is $\mathbf{U}_{p}$-closed and $(Y, \mathbf{U})$ is complete, then $\mu$ is $\mathbf{U}_{u}$-complete.

Theorem $3.15([6])$. If $\mu$ is a closed fuzzy set in $\left(Y^{X}, \mathbf{U}_{\mathbf{p}}\right)$ and for all $x \in X$, $\overline{e_{x}(\mu)}$ is complete in $(X, \mathbf{U})$, then the fuzzy set $\mu$ is complete in $\left(Y^{X}, \mathbf{U}_{u}\right)$.

Proposition 3.16. If $W_{\nu_{i}}, 1 \leq i \leq n$ are members of $\mathbf{U}_{u}$. Then the following hold:

i) $\bigwedge_{i=1}^{n} W_{\nu_{i}}=W_{\left(\bigwedge_{i=1}^{n} \nu_{i}\right)}$ and

ii) $W_{\nu}^{\alpha}=W_{\nu^{\alpha}}$, for $\alpha \in I_{1}$. 
Proof. i) For each $i, \mathrm{~W}_{\nu_{i}}=\bigwedge_{x \in X}\left(e_{x} \times e_{x}\right)^{-1}\left(\nu_{i}\right)$. Hence,

$$
\begin{aligned}
\bigwedge_{i=1}^{n} \mathrm{~W}_{\nu_{i}} & =\bigwedge_{i=1}^{n}\left\{\bigwedge_{x \in X}\left(e_{x} \times e_{x}\right)^{-1}\left(\nu_{i}\right)\right\} \\
& =\bigwedge_{x \in X}\left[\left(e_{x} \times e_{x}\right)^{-1}\left(\bigwedge_{i=1}^{n} \nu_{i}\right)\right. \\
& =\mathrm{W}_{\left(\bigwedge_{i=1}^{n} \nu_{i}\right)}
\end{aligned}
$$

ii) For each $\alpha \in I_{1}$,

$$
\begin{aligned}
\mathrm{W}_{\nu^{\alpha}} & =\bigcap_{x \in X}\left\{(f, g):(f(x), g(x)) \in \nu^{\alpha}\right\} \\
& =\bigcap_{x \in X}\left\{(f, g):\left(e_{x} \times e_{x}\right)(f, g) \in \nu^{\alpha}\right\} \\
& =\bigcap_{x \in X}\left\{(f, g):(f, g) \in\left(e_{x} \times e_{x}\right)^{-1}\left(\nu^{\alpha}\right)\right\} \\
& =\bigcap_{x \in X}\left\{(f, g):(f, g) \in\left[\left(e_{x} \times e_{x}\right)^{-1}(\nu)\right]^{\alpha}\right\} \\
& =\bigcap_{x \in X}\left[\left(e_{x} \times e_{x}\right)^{-1}(\nu)\right]^{\alpha} \\
& =\left[\bigwedge_{x \in X}\left(e_{x} \times e_{x}\right)^{-1}(\nu)\right]^{\alpha} \\
& =\left(\mathrm{W}_{\nu}\right)^{\alpha} .
\end{aligned}
$$

The following theorem shows that for each $\alpha \in I_{1}$, the $\alpha$-level uniformity of the fuzzy uniformity of uniform convergence $\mathbf{U}_{u}$ on the function space $\Im$ coincides with the uniformity of uniform convergence on $\Im$ when $Y$ is endowed with the uniformity $i_{u, \alpha}(\mathbf{U})$.

Theorem 3.17. For each $\alpha \in I_{1}, i_{u, \alpha}\left(\mathbf{U}_{u}\right)$ is the uniformity of uniform convergence on $\Im$, where $Y$ is endowed with the uniformity $i_{u, \alpha}(\mathbf{U})$.

Proof. Let $\mathrm{W}_{\nu}$ be a basic element for the fuzzy uniformity of uniform convergence $\mathbf{U}_{u}$, where $\nu \in \mathbf{U}$. Then $\mathbf{W}_{\nu}^{\alpha}$ is a basic element for the uniformity $i_{u, \alpha}\left(\mathbf{U}_{u}\right)$. By Proposition 3.16(ii), $\mathrm{W}_{\nu^{\alpha}}=\mathrm{W}_{\nu}^{\alpha}$ and the fact that $\mathrm{W}_{\nu^{\alpha}}$ is a basic element for the uniformity of uniform convergence on $\Im$, where $Y$ is endowed with the uniformity $i_{u, \alpha}\left(\mathbf{U}_{u}\right)$, the theorem follows.

Jäger in [9] showed that the notion of fuzzy uniformity of uniform convergence is a good extension. 
Theorem 3.18. Let $C(X, Y)$ denote the collection of all continuous functions from a topological space $X$ into a uniform space $Y$ and let $\mathcal{U}_{u}$ denote the uniformity of uniform convergence on $C(X, Y)$. Then the fuzzy topology associated with the fuzzy uniformity $\omega_{u}\left(\mathcal{U}_{u}\right)$ is jointly fuzzy continuous.

Proof. The uniform topology $T\left(\mathcal{U}_{u}\right)$ on $C(X, Y)$ is jointly continuous. Since in view of Theorem 2.8 the fuzzy topology of joint fuzzy continuity is a good extension, the fuzzy topology $\omega\left(T\left(\mathcal{U}_{u}\right)\right)$ is jointly fuzzy continuous. By Theorem 2.32, $\tau\left(\omega_{u}\left(\mathcal{U}_{u}\right)\right)=\omega\left(T\left(\mathcal{U}_{u}\right)\right)$. This shows that the fuzzy topology $\tau\left(\omega_{u}\left(\mathcal{U}_{u}\right)\right)$ associated with the fuzzy uniformity $\omega_{u}\left(\mathcal{U}_{u}\right)$ is jointly fuzzy continuous.

Corollary 3.19. Let $X$ be a topological space and let $Y$ be a uniform space with the uniformity $\mathcal{U}_{Y}$. Let $C_{f}(X, Y)$ denote the collection of all fuzzy continuous maps from the topologically generated fts $\omega(X)$ into the topologically generated $f t s \omega(Y)$. Then the fuzzy topology of uniform convergence on $C_{f}(X, Y)$ is jointly fuzzy continuous.

Proof. Since $X$ and $Y$ are topologically generated fts, $C_{f}(X, Y)$ and $C(X, Y)$ are equal as sets. Since the uniformity of uniform convergence is a good extension, the fuzzy uniformity of uniform convergence $\mathbf{U}_{u}$ is same as $\omega_{u}\left(\mathcal{U}_{u}\right)$, where $\mathcal{U}_{u}$ is the uniformity of uniform convergence on $C(X, Y)$. Hence in view of Theorem 3.18, the fuzzy topology associated with the fuzzy uniformity of uniform convergence $\mathbf{U}_{u}$ is jointly fuzzy continuous.

Definition 3.20. Let $X$ be a fuzzy topological space and let $(Y, \mathbf{U})$ be a fuzzy uniform space. Let $\mathcal{S}$ be the collection of all starplus-compact fuzzy sets in $X$. For each $\kappa \in \mathcal{S}$ and $\nu \in \mathbf{U}$, define a fuzzy set $W(\kappa, \nu): \Im \times \Im \longrightarrow I$ by

$$
\begin{aligned}
W(\kappa, \nu)(f, g) & =\bigwedge_{x \in \text { supp }}\left(e_{x} \times e_{x}\right)^{-1}(\nu)(f, g) \\
& =\bigwedge_{x \in \text { supp } \kappa} \nu(f(x), g(x)) .
\end{aligned}
$$

Then the collection of all fuzzy sets $\{W(\kappa, \nu): \kappa \in \mathcal{S}, \nu \in \mathbf{U}\}$ is a base for a fuzzy uniformity on $\Im$ and is called the fuzzy uniformity of uniform convergence on starplus-compacta.

Theorem 3.21. Let $\Im$ be the set of all fuzzy continuous maps from a topologically generated fts $\left(X, \tau_{X}\right)$ into a fuzzy uniform space $(Y, \mathbf{U})$. Then the fuzzy topology of fuzzy uniform convergence on starplus-compacta is the starpluscompact open fuzzy topology.

Proof. Let $\mathbf{U}_{* C}^{+}$be the fuzzy uniformity of uniform convergence on starpluscompacta. Since $X$ is a topologically generated fts, a subset $K$ of $X$ is compact in $\left(X, i_{0}\left(\tau_{X}\right)\right)$ if and only if $\chi_{K}$ is starplus-compact in $\left(X, \tau_{X}\right)$. Hence $i_{u \alpha}\left(\mathbf{U}_{* C}^{+}\right)=\mathcal{U}_{U C}$, where $\mathcal{U}_{U C}$ denotes the uniformity of uniform convergence on compacta, where $X$ is endowed with the topology $i_{0}\left(\tau_{X}\right)$ and $Y$ is equipped with the uniformity $i_{u \alpha}(\mathbf{U})$. So by [11, Theorem 7.11], $T\left(i_{u \alpha}\left(\mathbf{U}_{* C}^{+}\right)\right)$is the compact open topology for each $\alpha \in I_{1}$. By Theorem 2.6, $T\left(i_{u \alpha}\left(\mathbf{U}_{* C}^{+}\right)\right)=i_{\alpha}\left(\tau_{* C}^{+}\right)$ 
for each $\alpha \in I_{1}$. Again by Theorem 2.34, $i_{\alpha}\left(\tau\left(\mathbf{U}_{* C}^{+}\right)\right)=T\left(i_{u \alpha}\left(\mathbf{U}_{* C}^{+}\right)\right)=i_{\alpha}\left(\tau_{* C}^{+}\right)$ for each $\alpha \in I_{1}$. This completes the proof.

\section{Fuzzy EquiCONTINUity}

In this section we introduce the notion of fuzzy equicontinuity and fuzzy uniform equicontinuity on fuzzy subsets of $\Im$ and obtain results, which show that if a fuzzy subset $\kappa$ of $Y^{X}$ is fuzzy equicontinuous (respectively, fuzzy uniformly equicontinuous), then each $f \in \operatorname{supp} \kappa$ is fuzzy continuous (respectively, fuzzy uniformly continuous).

Definition 4.1. Let $(X, \tau)$ be a fts and let $(Y, \mathbf{U})$ be a fuzzy uniform space. Then a fuzzy subset $\kappa$ of $Y^{X}$ is said to be fuzzy equicontinuous at a fuzzy point $x_{\alpha}$ of $X$ if for each $\mu \in \mathbf{U}$, there is a $\tau$-neighbourhood $\eta$ of $x_{\alpha}$ such that $f(\eta) \leq \mu\left\langle f\left(x_{\alpha}\right)\right\rangle$ for each $f \in$ supp $\kappa$. We say that the fuzzy subset $\kappa$ of $Y^{X}$ is fuzzy equicontinuous on a fuzzy subset $\theta$ of $X$ if $\kappa$ is fuzzy equicontinuous at each fuzzy point $x_{\alpha}$ in $\theta$.

Definition 4.2. A fuzzy subset $\kappa$ of $Y^{X}$, where $\left(X, \mathbf{U}_{X}\right)$ and $\left(Y, \mathbf{U}_{Y}\right)$ are fuzzy uniform spaces, is said to be fuzzy uniformly equicontinuous if

$$
\bigwedge_{f \in \text { supp } \kappa}(f \times f)^{-1}(\mu) \in \mathbf{U}_{X} \text { for each } \mu \in \mathbf{U}_{Y} .
$$

The following is a characterization of fuzzy equicontinuity.

Theorem 4.3. A fuzzy subset $\kappa$ of $Y^{X}$ is fuzzy equicontinuous if and only if for each $\mu \in \mathbf{U}_{Y}$, the fuzzy set $\bigwedge_{f \in \text { supp } \kappa} f^{-1}\left(\mu\left\langle f\left(x_{\alpha}\right)\right\rangle\right)$ is a neighbourhood of $x_{\alpha}$.

Proof. Suppose that a fuzzy subset $\kappa$ of $Y^{X}$ is fuzzy equicontinuous at a fuzzy point $x_{\alpha}$ of $X$. Then for each $\mu \in \mathbf{U}_{Y}$, there is a $\tau$-neighbourhood $\eta$ of $x_{\alpha}$ such that $f(\eta) \leq \mu\left\langle f\left(x_{\alpha}\right)\right\rangle$ for each $f \in$ supp $\kappa$. So $\eta \leq \bigwedge_{f \in \text { supp } \kappa} f^{-1}\left(\mu\left\langle f\left(x_{\alpha}\right)\right\rangle\right)$ and hence $\bigwedge_{f \in \text { supp } \kappa} f^{-1}\left(\mu\left\langle f\left(x_{\alpha}\right)\right\rangle\right)$ is a neighbourhood of $x_{\alpha}$.

Conversely, suppose that $\bigwedge_{f \in \text { supp } \kappa} f^{-1}\left(\mu\left\langle f\left(x_{\alpha}\right)\right\rangle\right)$ is a neighbourhood of the fuzzy point $x_{\alpha}$ for each $\mu \in \mathbf{U}_{Y}$. Let $\eta=\bigwedge_{f \in \text { supp } \kappa} f^{-1}\left(\mu\left\langle f\left(x_{\alpha}\right)\right\rangle\right)$. Then clearly $g(\eta) \leq \mu\left\langle g\left(x_{\alpha}\right)\right\rangle$ for each $g \in \operatorname{supp} \kappa$ and so $\kappa$ is fuzzy equicontinuous at the fuzzy point $x_{\alpha}$.

Theorem 4.4. If a fuzzy set $\kappa$ in $Y^{X}$ is fuzzy equicontinuous, then supp $\kappa$ is equicontinuous, where $X$ is endowed with the topology $i_{0}\left(\tau_{X}\right)$ and $Y$ is equipped with the uniformity $i_{u, \alpha}\left(\mathbf{U}_{Y}\right)$.

Proof. Let $\kappa$ be fuzzy equicontinuous. Then for each fuzzy point $x_{\lambda}$ in $X$ and for each $\mu \in \mathbf{U}_{Y}$, there exist a neighbourhood $\eta$ of $x_{\lambda}$ in $X$ such that $f(\eta) \leq \mu\left\langle f\left(x_{\lambda}\right)\right\rangle$ for each $f \in$ supp . Hence in particular, $f(\eta) \leq \mu\left\langle f\left(x_{1}\right)\right\rangle$ with $\lambda=1$. This shows that $f\left(\eta^{\beta}\right) \subset \mu^{\beta}\langle f(x)\rangle, \beta \in[0,1-\alpha)$. Thus for each 
$x \in X$ and $\mu^{\beta} \in i_{u, \alpha}\left(\mathbf{U}_{Y}\right)$, there exist a neighbourhood $\eta^{\beta}$ of $x$ such that $f\left(\eta^{\beta}\right) \subset \mu^{\beta}\langle f(x)\rangle$, for each $f \in$ supp $\kappa$. Hence supp $\kappa$ is equicontinuous.

Theorem 4.5. If a fuzzy subset $\kappa$ in $Y^{X}$ is fuzzy equicontinuous, then $\kappa^{\alpha}$ is equicontinuous for each $\alpha \in I_{1}$, where $Y$ is equipped with the uniformity $i_{u, \alpha}\left(\mathbf{U}_{Y}\right)$.

First we prove the following lemma.

Lemma 4.6. If $\nu \leq \kappa$ and $\kappa$ is fuzzy equicontinuous then $\nu$ is also fuzzy equicontinuous.

Proof. Since $\nu \leq \kappa$, supp $\nu \subset$ supp $\kappa$.

Hence $\bigwedge_{f \in \text { supp } \kappa} f^{-1}\left(\mu\left\langle f\left(x_{\alpha}\right)\right\rangle\right) \leq \bigwedge_{f \in \text { supp }} f^{-1}\left(\mu\left\langle f\left(x_{\alpha}\right)\right\rangle\right)$ and so the result follows.

Proof of Theorem 4.5. Since $\kappa^{\alpha} \subset$ supp $\kappa$ for each $\alpha \in I_{1}$ and since $\kappa$ is fuzzy equicontinuous then by Theorem 4.4 and Lemma $4.6, \kappa^{\alpha}$ is equicontinuous. $\square$.

Theorem 4.7. If a fuzzy subset $\kappa$ of $Y^{X}$ is fuzzy equicontinuous, then each $f \in$ suppк is fuzzy continuous.

Proof. Since $\bigwedge_{f \in \text { supp } \kappa} f^{-1}\left(\mu\left\langle f\left(x_{\alpha}\right)\right\rangle\right) \leq f^{-1}\left(\mu\left\langle f\left(x_{\alpha}\right)\right\rangle\right)$ for each fuzzy point $x_{\alpha}$ of $X, f^{-1}\left(\mu\left\langle f\left(x_{\alpha}\right)\right\rangle\right)$ is a neighbourhood of $x_{\alpha}$ for each $\mu \in \mathbf{U}_{Y}$ and so $f$ is fuzzy continuous at each $x_{\alpha}$. Hence $f$ is fuzzy continuous on $X$.

Theorem 4.8. If $\kappa$ is fuzzy uniformly equicontinuous, then each $f \in$ supp $\kappa$ is fuzzy uniformly continuous.

Proof. Suppose that $\kappa$ is fuzzy uniformly equicontinuous. Then for each $\mu \in$ $\mathbf{U}_{Y}, \bigwedge_{f \in \text { supp }}(f \times f)^{-1}(\mu) \in \mathbf{U}_{X}$. This implies that for each $\mu \in \mathbf{U}_{Y}$, $(f \times f)^{-1}(\mu) \in \mathbf{U}_{X}$. Hence $f$ is fuzzy uniformly continuous.

Acknowledgements. The authors are thankful to the referee for helpful suggestions.

\section{REFERENCES}

[1] I. W. Alderton, Function spaces in fuzzy topology, Fuzzy Sets and Systems 32 (1989), 115-124.

[2] C. Arzelà, Funzioni di Linee, Atti della Reale Accademia dei Lincei, Rendiconti 5 (1889), 342-348.

[3] G. Ascoli, Le Curve Limite di una Varieta Data di Curve, Mem. Acad. Lincei (3) 18(1883), $512-586$. 
[4] N. Bourbaki, Topologe géneralé, Ch.I et II, Paris, 1940.

[5] M. H. Burton, Cauchy filters and Prefilters, Fuzzy Sets and Systems 54 (1993), 317-331.

[6] M. H. Burton, The fuzzy uniformisation of function spaces, Quaestiones Math. 20 (1997), 283-290.

[7] R. H. Fox, On topologies for function spaces, Bull. Amer. Math. Soc. 51 (1945), 429-432.

[8] J. Hadamard, Sur Certaines Applications Possibles de la Théorie des Ensembles, Verhandlungen des Ersten Internationalen der Mathematiker-Kongresses, B.G.Teubner, Leipzig (1898).

[9] G. Jäger, Fuzzy uniform convergence and equicontinuity, Fuzzy Sets and Systems 109 (2000), 187-198.

[10] A. Kandil, Khaled A. Hashem and Nehad N. Morsi, A level topologies criterian for Lowen fuzzy uniformity, Fuzzy Sets and Systems 62 (1994), 211-226.

[11] J. L. Kelley, General Topology, Van Nosterand, New York, 1955.

[12] J. K. Kohli and A. R. Prasannan, Fuzzy topologies on function spaces, Fuzzy Sets and Systems 116 (3)(2000), 415-420.

[13] J. K. Kohli and A. R. Prasannan, Starplus-compactness in fuzzy topological spaces and starplus-compact open fuzzy topologies on function spaces, J. Math. Anal. Appl. 254 (2001), 87-100.

[14] R. Lowen, Fuzzy topological spaces and fuzzy compactness, J. Math. Anal. Appl. 56 (1976), 621-633.

[15] R. Lowen, Convergence in fuzzy topological spaces, Gen. Top. Appl. 10 (1979), 147-160.

[16] R. Lowen, Fuzzy uniform spaces, J. Math. Anal. Appl. 82 (1981), 370-385.

[17] Y. W. Peng, Topological structure of a fuzzy function space- the pointwise convergent topology and compact open topology, Kexue Tongbao (English Ed.) 29 (1984), 289-292.

[18] A.Weil, Sur les Espaces à Structure Uniform et sur la Topologie Générale, Act.Sci.et Ind. 551, Hermann, Paris (1937).

RECEIVED APRIL 2005

AcCePted March 2006

\section{J. K. KoHLI}

Department of Mathematics, Hindu College, University of Delhi, Delhi - 110 007, India.

\section{A. R. Prasannan (arprasannan@yahoo.co.in)}

Department of Mathematics, Maharaja Agrasen College, University of Delhi, Pocket - IV, Mayur Vihar - I, Delhi - 110 091, India. 